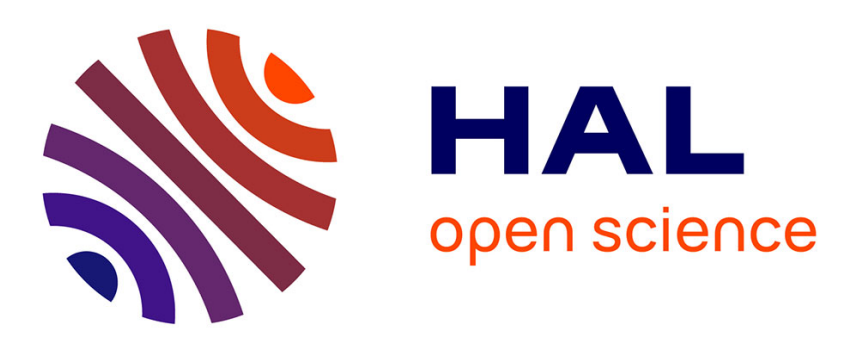

\title{
Mesures de constantes diélectriques complexes à l'aide d'un dispositif autonome utilisant un calculateur compatible PC
}

\author{
M. Craman, J. David, R. Crampagne
}

\section{- To cite this version:}

M. Craman, J. David, R. Crampagne. Mesures de constantes diélectriques complexes à l'aide d'un dispositif autonome utilisant un calculateur compatible PC. Revue de Physique Appliquée, 1990, 25 (5), pp.469-474. 10.1051/rphysap:01990002505046900 • jpa-00246207

\section{HAL Id: jpa-00246207 https://hal.science/jpa-00246207}

Submitted on 1 Jan 1990

HAL is a multi-disciplinary open access archive for the deposit and dissemination of scientific research documents, whether they are published or not. The documents may come from teaching and research institutions in France or abroad, or from public or private research centers.
L'archive ouverte pluridisciplinaire HAL, est destinée au dépôt et à la diffusion de documents scientifiques de niveau recherche, publiés ou non, émanant des établissements d'enseignement et de recherche français ou étrangers, des laboratoires publics ou privés. 


\title{
Mesures de constantes diélectriques complexes à l'aide d'un dispositif autonome utilisant un calculateur compatible PC
}

\author{
M. Craman, J. David et R. Crampagne \\ E.N.S.E.E.I.H.T., Laboratoire d'Electronique, 2, rue Camichel, 31071 Toulouse Cedex, France \\ (Reçu le 10 juillet 1989, révisé le 13 novembre 1989 et le 3 janvier 1990, accepté le 3 janvier 1990)
}

\begin{abstract}
Résumé. - La mesure de constantes diélectriques est effectuée à l'aide d'un oscillateur, comportant un résonateur coaxial ouvert. Cette sonde active est reliée à un micro-ordinateur type PC, par l'intermédiaire d'une carte spécifique à cette application. Elle permet, à partir de la mesure de la fréquence et de l'amplitude d'oscillation, de déterminer la permittivité diélectrique des matériaux étudiés.
\end{abstract}

\begin{abstract}
Measure of dielectric coefficients is carried out with an oscillator, involving an open end transmission line resonator. This active probe is connected to a PC computer, through the agency of a specific numerical board. It permits, from the measure of frequency and amplitude of oscillation, to determine the complex permittivity of studied materials.
\end{abstract}

\section{Introduction.}

Les mesures de constantes diélectriques ont, depuis fort longtemps, fait l'objet d'études approfondies. Les méthodes, pour y parvenir, se sont souvent avérées délicates à mettre en œuvre, car nécessitant un matériel sophistiqué et coûteux. La solution présentée ici s'affranchit de ces difficultés, grâce principalement à l'usage d'une sonde active, d'une carte spécifique et d'un calculateur compatible PC (Fig. 1).

\section{Principe de la méthode.}

La méthode repose sur la perturbation du champ électromagnétique à l'ouverture d'une ligne coaxiale, par un matériau inconnu (Fig. 2). Cette modification du champ, au voisinage de la discontinuité, peut être modélisée, à l'entrée de la ligne, par une capacité $C$ et une conductance $G$ en parallèle $[1,2]$. Pour la suite des calculs, on tiendra compte des hypothèses suivantes :

- les milieux ne comprennent ni charge, ni courant ;

- les dimensions transversales de la discontinuité sont petites devant la longueur d'onde employée ;

- aux fréquences utilisées, seul le mode TEM peut se propager.

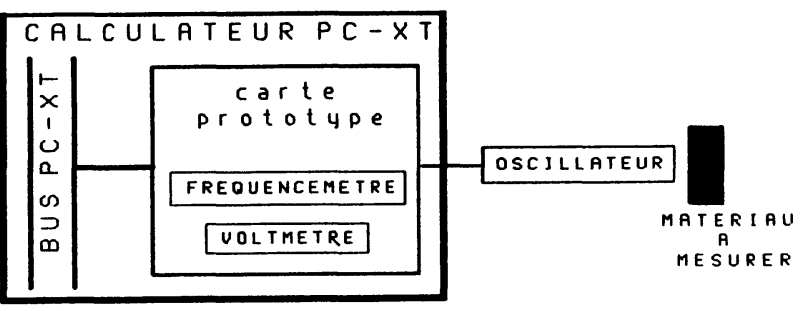

Fig. 1. - Montage de la manipulation réalisée.

[Assembly line of manipulation.]

Le champ électrique vérifie l'équation suivante : $E=-\operatorname{grad} V$, avec $V=V v$ sur le conducteur central et $V=0$ sur le conducteur extérieur (Fig. 3).

A l'interface nous avons la relation suivante :

$$
\varepsilon_{1}^{\prime} \cdot \frac{\mathrm{d} V}{\mathrm{~d} z}=\varepsilon_{2}^{\prime} \cdot \frac{\mathrm{d} V}{\mathrm{~d} z}
$$

Au cours d'une période, l'énergie électrique emmagasinée dans les 2 régions a pour valeur moyenne :

$$
W_{\mathrm{e}}=\frac{1}{4} \cdot \varepsilon_{1}^{\prime} \cdot \int_{v_{1}}|E|^{2} \cdot \mathrm{d} v+\frac{1}{4} \cdot \varepsilon_{2}^{\prime} \cdot \int_{v_{2}}|E|^{2} \mathrm{~d} v
$$

$v_{1}$ et $v_{2}$ sont respectivement les volumes considérés dans les régions 1 et 2 . 


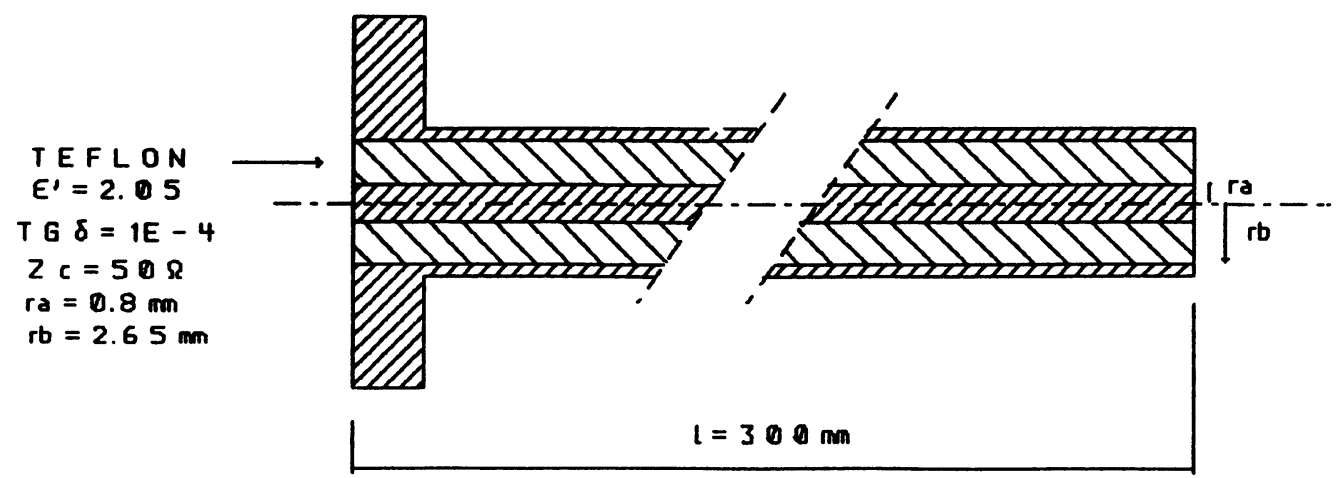

Fig. 2. - Description de la ligne coaxiale.

[Transmission line resonator.]

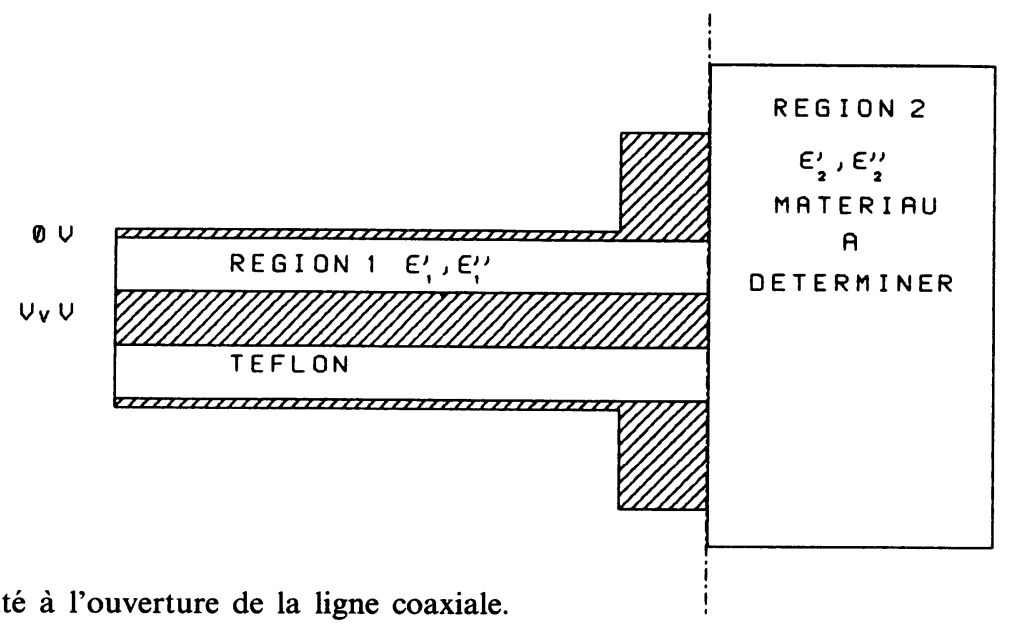

Fig. 3. - Discontinuité à l'ouverture de la ligne coaxiale.

[Discontinuity at the aperture of the coaxial line.]

Si le champ électrique était uniforme, l'énergie électrique aurait pour valeur moyenne :

$$
W_{\mathrm{e}}^{\prime}=\frac{1}{4} \cdot C_{1} \cdot \mathrm{d} \ell_{1} \cdot V v^{2}
$$

où $C_{1}$ est la capacité linéique de la ligne dans le cas non perturbé, $\mathrm{d} \ell_{1}$ la longueur de ligne considérée. On a: $W_{\mathrm{e}}>W_{\mathrm{e}}^{\prime}$. Cette différence d'énergie peut se traduire par une réactance capacitive, placée en parallèle dans le plan de discontinuité

$$
\begin{array}{r}
C=\frac{1}{V v^{2}} \cdot\left[\varepsilon_{1}^{\prime} \cdot \int_{v_{1}}|E|^{2} \cdot \mathrm{d} v+\varepsilon_{2}^{\prime} \cdot\right. \\
\left.\int_{v_{2}}|E|^{2} \cdot \mathrm{d} v\right]- \\
-C_{1} \cdot \mathrm{d} \ell_{1} .
\end{array}
$$

D'une manière similaire, la différence d'énergie moyenne dissipée se traduit par une conductance, qui a pour expression :

$$
\begin{array}{r}
G=\frac{w}{V v^{2}} \cdot\left[\varepsilon_{1}^{\prime \prime} \cdot \int_{v_{1}}|E|^{2} \cdot \mathrm{d} v+\varepsilon_{2}^{\prime \prime} \cdot \int_{v_{2}}|E|^{2} \cdot \mathrm{d} v\right]- \\
-G_{1} \cdot \mathrm{d} \ell_{1}
\end{array}
$$

où $G_{1}$ est la conductance linéique de la ligne dans le cas non perturbé. La résolution de ces intégrales fait appel à des méthodes numériques décrites dans [1] et [4] et permettent d'établir que $C\left(\varepsilon^{\prime}\right)$ est une droite affine.

L'admittance de la discontinuité d'écrit :

$$
Y=G+j \cdot C \cdot \cdots \text {. }
$$

Ainsi mise en évidence, cette admittance est ramenée par la ligne coaxiale à l'entrée de l'oscillateur (Fig. 4) sous la forme d'une impédance de la forme :

$$
Z=R_{0}+j \cdot\left(L w-1 /\left(C_{0} \cdot w\right)\right) .
$$

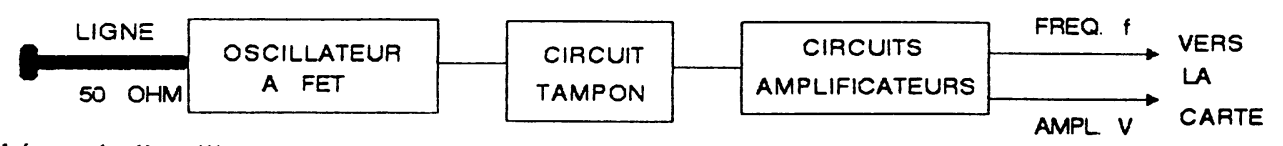

Fig. 4. - Schéma de l'oscillateur.

[Oscillator scheme.] 


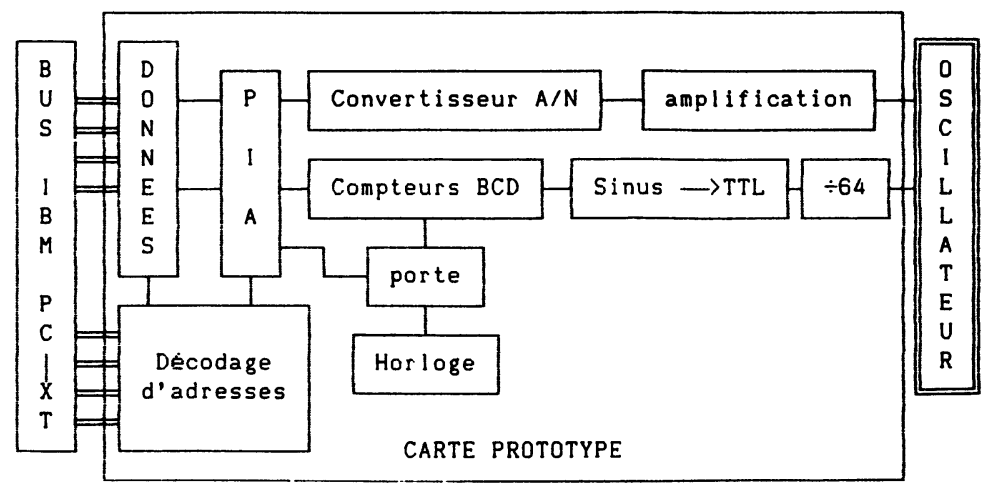

Fig. 5. - Bloc synoptique de la carte numérique.

[Block diagram of the electronic board.]

Cet oscillateur va voir alors ses paramètres modifiés, et donc sa fréquence et son amplitude d'oscillation.

L'acquisition de ces variations se fait par l'intermédiaire de la carte intégrée au calculateur (Fig. 5), constituée d'un voltmètre et d'un fréquencemètre.

Le problème consiste à créer un lien entre ces variations $(\mathrm{d} f$ et $\mathrm{d} V$ ) et les caractéristiques diélectriques du matériau placé en bout de ligne. Pour y parvenir, il n'est pas envisageable d'effectuer la modélisation du dispositif de mesure. En effet, les éléments du montage présentent des dispersions, en particulier au niveau des caractéristiques des FET utilisés ; il faudrait alors faire une nouvelle modélisation pour chaque montage réalisé. Il est préférable de s'orienter vers un étalonnage du dispositif à l'aide d'une banque d'échantillons connus, couvrant le domaine à étudier. Pour accomplir cette initialisation, il est nécessaire d'examiner les paramètres dépendants du matériau $\left(R_{0}, L, C_{0}\right)$.

\section{Détermination des paramètres.}

Ce système, chargé par un diélectrique, correspond à un montage Clapp (Fig. 6). On obtient les condi-

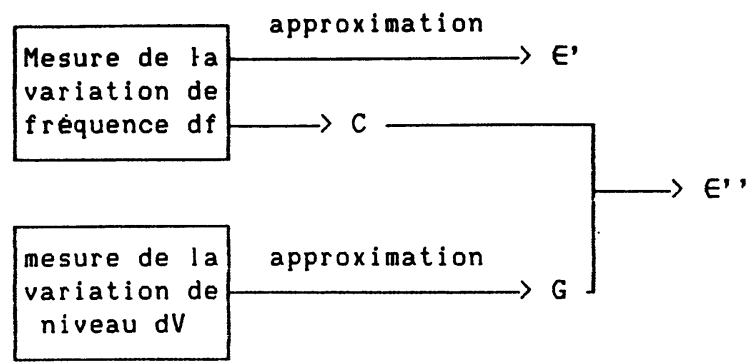

Fig. 6. - Schéma équivalent de l'oscillateur.

[Equivalent scheme of the oscillator.]

tions d'oscillation :

$$
L=\frac{C_{1} \cdot C_{2}+C_{0} \cdot\left(C_{1}+\left(1+R_{0} / R\right) \cdot C_{2}\right)}{C_{0} \cdot C_{1} \cdot C_{2} \cdot w_{2}}
$$

avec $w=2 \cdot \pi \cdot f$ où $f$ est la fréquence d'oscillation

$$
\begin{aligned}
\left(R \cdot R_{0} \cdot C_{0} \cdot C_{1} \cdot C_{2}+L \cdot C_{0} \cdot C_{2}\right) \times \\
\quad \times w^{2} \leqslant C_{2}+C_{0}+R \cdot g m \cdot C_{0} .
\end{aligned}
$$

La théorie du calcul sur les lignes donne :

$$
\begin{gathered}
\frac{L \cdot C_{0} \cdot w^{2}-1}{C_{0} \cdot w}=Z_{\mathrm{c}} \cdot \frac{\operatorname{tg}(\beta \cdot \ell) \cdot\left[Z_{\mathrm{c}}^{2} \cdot C^{2} \cdot w^{2} \cdot\left(1+\operatorname{tg}^{2} \delta\right)-1\right]+Z_{\mathrm{C}} \cdot C \cdot w \cdot\left[\operatorname{tg}^{2}(\beta \cdot \ell)-1\right]}{\left(Z_{\mathrm{c}} \cdot C \cdot w \cdot \operatorname{tg} \delta\right)^{2}+\left[\operatorname{tg}(\beta \cdot \ell)+Z_{\mathrm{c}} \cdot C \cdot w\right]^{2}} \\
R_{0}=\frac{Z_{\mathrm{c}}^{2} \cdot G \cdot\left(1+(\operatorname{tg}(\beta \cdot \ell))^{2}\right)}{\left(Z_{\mathrm{c}} \cdot G\right)^{2}\left[\operatorname{tg}(\beta \cdot \ell)+C \cdot w \cdot Z_{\mathrm{c}}\right]^{2}}
\end{gathered}
$$

avec $\beta=\frac{2 \cdot \pi}{\lambda}$ où $\lambda$ est la longueur d'onde $\lambda=\frac{3 \times 10^{8}}{\sqrt{2,05} \cdot f} m$, le Téflon étant milieu de propagation de la ligne ;

$\ell$ : longueur de la ligne $(300 \mathrm{~mm})$.

$Z_{\mathrm{c}}$ : impédance caractéristique de la ligne $(50 \mathrm{ohm})$.
Compte tenu de la gamme de matériaux mesurables par cette méthode, $C$ a une valeur comprise entre une fraction de picofarad et quelques picofarads. D'autre part, $\operatorname{tg}(\beta .1) \approx-3$ et $Z_{\mathrm{c}} \cdot G \ll 1$; (8) peut donc s'écrire :

$$
R_{0} \approx Z_{\mathrm{c}^{2}} \cdot G
$$


La ligne, ouverte, considérée sans pertes, ramène à l'entrée du circuit oscillant une réactance $X_{0}$ déterminée par:

$$
X_{0}=-\frac{Z_{\mathrm{c}}}{\operatorname{tg}\left(\beta_{0} \cdot \ell\right)} \quad \text { avec } \quad \beta_{0}=\frac{2 \pi}{\lambda_{0}}
$$

$R_{0}$ restant faible devant $R$ (résistance d'entrée d'un
FET), (5) devient alors :

$$
X=\frac{L \cdot C_{0} \cdot w^{2-1}}{C_{0} \cdot w} \approx \frac{C_{1}+C_{2}}{C_{1} \cdot C_{2} \cdot w}=\frac{A}{f}
$$

avec $A=\left(C_{1}+C_{2}\right) /\left(2 \cdot \pi \cdot C_{1} \cdot C_{2}\right)$ et $X_{0}=$ $A / f_{0}\left(f_{0}\right.$ fréquence d'oscillation à vide; $f_{0}=$ $207,8 \mathrm{MHz})$.

L'angle de perte du matériau étudié restant limité ( $\operatorname{tg} \delta<0,5$ ), la relation (7) se simplifie, et on peut la mettre sous la forme :

$$
X \approx Z_{\mathrm{c}} \cdot \frac{\left[\operatorname{tg}(\beta \cdot \ell)+Z_{\mathrm{c}} \cdot C \cdot w\right] \cdot\left[\operatorname{tg}(\beta \cdot \ell) \cdot Z_{\mathrm{c}} \cdot C \cdot w-1\right]}{\left[\operatorname{tg}(\beta \cdot \ell)+Z_{\mathrm{c}} \cdot w\right]^{2}} .
$$

On obtient $C$ :

$$
C=\frac{X \cdot \operatorname{tg}(\beta \cdot \ell)+Z_{\mathrm{c}}}{\left[Z_{\mathrm{c}} \cdot \operatorname{tg}(\beta \cdot \ell)-X\right] \cdot Z_{\mathrm{c}} \cdot w}
$$

$C$ est parfaitement déterminé et ne dépend que de $f$, aux approximations près. Puisque $C$ varie linéairement avec $\varepsilon^{\prime}$, on peut effectuer lors de l'étalonnage une étude directe de la courbe $\varepsilon^{\prime}(\mathrm{d} f)$ à l'aide d'une méthode d'approximation (moindres carrés).

D'autre part, à partir de l'analyse du schéma équivalent simplifié du montage (Fig. 6), la tension à l'entrée du montage peut s'exprimer sous la forme suivante:

$$
V_{\mathrm{e}}=\frac{V_{\mathrm{gs}} \cdot\left[R_{0} \cdot g m-2 \cdot \pi \cdot A \cdot C_{2}+j \cdot\left(R_{0} \cdot C_{2} \cdot w+g m \cdot X\right)\right]}{1-2 \cdot \pi \cdot A \cdot C_{2}+j \cdot R_{0} \cdot C_{2} \cdot w} .
$$

Le module de $V_{\mathrm{e}}$ (et donc l'amplitude du signal à la sortie du montage) dépend pour l'essentiel de la valeur de $R_{0}$ qui varie proportionnellement à $G$, car $\mathrm{d} f \leqslant 2 \times f_{0} / 100$ pour le domaine de mesure qui nous intéresse ici.

Il est ainsi possible de tracer une courbe d'étalonnage $G(\mathrm{~d} V)$ par l'intermédiaire de la relation $G=C \cdot w \cdot \operatorname{tg} \delta, \operatorname{tg} \delta$ étant connu pour les matériaux de référence.

\section{Principe de mesure.}

Un étalonnage préalable est effectué, à l'aide de matériaux connus (banque d'échantillons de référence), afin de déterminer les paramètres qui permettent de caractériser les diélectriques.

L'initialisation se fait ainsi :

- l'inconnue à déterminer dans un premier temps est $A$. Afin d'y parvenir, on mesure la fréquence $f_{0}$ et l'amplitude $V_{0}$ du signal pour la ligne à vide.

- on mesure la fréquence $f$ et l'amplitude $V$ d'oscillation, pour chaque référence placée en bout de ligne, que l'on compare à la fréquence $f_{0}$ et l'amplitude $V_{0}$ d'oscillation à vide. On peut alors calculer $C$ par l'intermédiaire des équations écrites précédemment et déterminer $G$ par la relation :

$G=C \cdot w \cdot \operatorname{tg} \delta$ où $\operatorname{tg} \delta$ représente les pertes des matériaux étalons.

Il est possible alors de tracer $\varepsilon^{\prime}$ et $G$ en fonction respectivement de la variation de fréquence et de la variation d'amplitude.

Une fois l'étalonnage effectué, il suffit de placer la sonde sur les matériaux à caractériser. Les variations

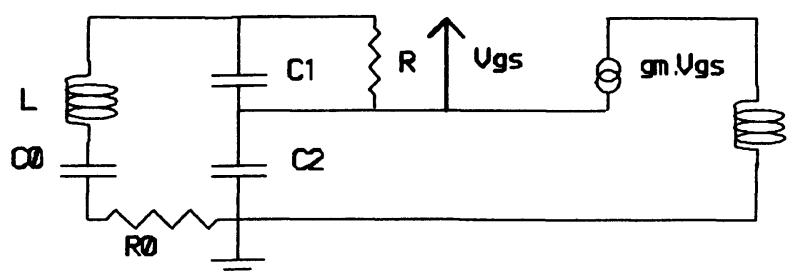

Fig. 7. - Descriptif de la méthode de résolution.

[Description of the processing.]

d'amplitude et de fréquence permettent de connaître $\varepsilon^{\prime}$ et $G$ par approximation. Connaissant $C$ par le calcul (11), on en déduit alors $\operatorname{tg} \delta$, et ainsi $\varepsilon^{\prime \prime}$ (Fig. 7). 


\section{Domaines d'application.}

La méthode a été validée expérimentalement pour des matériaux de caractéristiques :

$-\varepsilon^{\prime} \leqslant 20$

$-\varepsilon^{\prime \prime} \leqslant 1$.

Le tableau (Fig. 8) donne quelques exemples d'échantillons mesurés, n'ayant pas participé à l'étalonnage.

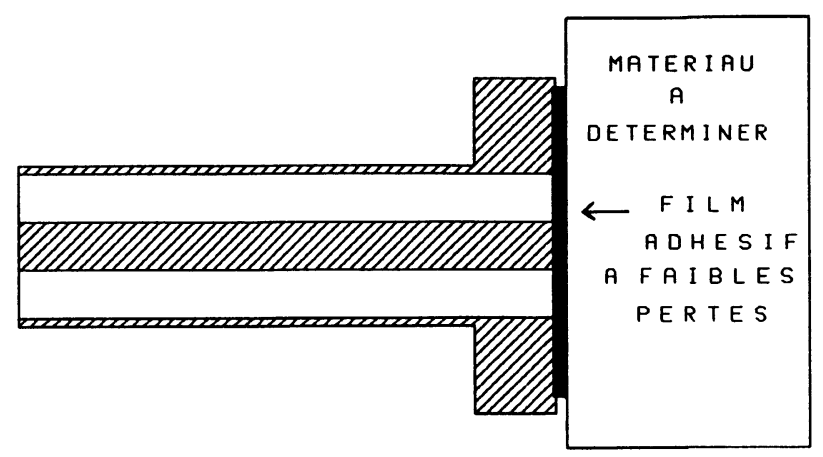

Fig. 8. - Montage pour des valeurs élevées de la permittivité.

[Arrangement for high values of permittivity.]

D'autres mesures de matériaux à pertes, disponibles au laboratoire et caractérisés par ailleurs, ont permis de vérifier le bien-fondé de la méthode.

Ce système de mesure présente une sensibilité élevée sur la détermination de $\varepsilon^{\prime}$, mise en évidence par la caractérisation possible du polystyrène expansé, dont la constante diélectrique se trouve très proche de 1 . Ce résultat est dû à la très bonne

Tableau I. - Tableau des résultats expérimentaux. [Experimental results.]

\begin{tabular}{|c|c|c|c|c|}
\hline Matériaux & \multicolumn{2}{|c|}{$\begin{array}{c}\text { Publiés ou mesurés } \\
\text { par ailleurs }\end{array}$} & \multicolumn{2}{|c|}{ mesurés } \\
$f_{0}=207,8 \mathrm{MHz}$ & $\varepsilon^{\prime} / \varepsilon_{0}$ & $\operatorname{tg} \delta$ & $\varepsilon^{\prime} / \varepsilon_{0}$ & $\operatorname{tg} \delta$ \\
\hline Air & 1,0 & 0 & 1,0 & 0 \\
Dépron & 1,03 & $<1 e-4$ & 1,05 & 0 \\
Téflon & 2,05 & $1 e-4$ & 2,13 & 0 \\
Plexiglass & 2,65 & $6 e-3$ & 2,61 & $3 e-3$ \\
Bois & 2,5 & $7 e-2$ & 2,4 & $5 e-2$ \\
Alumine & 9 & $<1 e-4$ & 9,3 & 0 \\
Silicium & 12 & 0,2 & 11,5 & 0,15 \\
\hline
\end{tabular}

stabilité en fréquence de l'oscillateur, et la précision présentée par le fréquencemètre numérique.

Compte-tenu des expérimentations effectuées, il est apparu que la plage de mesure de $\varepsilon^{\prime}$ est étendue : il semble possible de déterminer des matériaux dont la permittivité diélectrique relative est de l'ordre de 50.

Par contre, un manque de sensibilité concernant les pertes a été mis en évidence lors des essais. Ainsi, il est difficile de trouver la valeur de $\operatorname{tg} \delta$ pour des diélectriques à pertes faibles, pratiquement indétectables par le système de mesure, ou fortes, qui ne garantissent plus les conditions d'oscillation (5) et (6), auquel cas l'oscillateur cesse de fonctionner.

La gamme utile de mesure se situe dans l'intervalle $0,05<\operatorname{tg} \delta<0,5$.

\section{Conditions d'application.}

Cette méthode permet, théoriquement, la mesure des solides, des liquides et, éventuellement, des gaz.

Il est inutile de donner aux échantillons une forme précise par un usinage rigoureux. La seule contrainte est qu'ils doivent présenter une surface plane, avec un bon état de surface, permettant d'assurer un bon contact avec la sonde.

D'autre part, afin que la mesure soit correcte, il est souhaitable que l'élément à étudier présente un volume au moins égal à une hémisphère de diamètre 2 . $r b$; cette précaution permet aux lignes de champ de rester confinées à l'intérieur du matériau. Dans le cas contraire, l'initialisation est effectuée avec des diélectriques de même épaisseur que les échantillons à caractériser.

La méthode repose sur une approximation. Il en résulte qu'il faut éviter d'explorer une étendue de valeurs trop vaste pour un seul étalonnage, qui donnerait alors de mauvais résultats. Il est préférable, dans ce cas, de fractionner le domaine d'étude. Il est alors impératif de situer les matériaux à déterminer dans la bonne plage de mesure. Pour des diélectriques à constante très élevée, les conditions d'oscillation (5) et (6) peuvent ne plus être remplies et entraîner un arrêt de l'oscillateur. On peut s'affranchir de ces difficultés en plaçant en bout de ligne un film adhésif mince, à faibles pertes, qui diminuera la sensibilité de la sonde en ajoutant de la sorte une capacité série, mais étendra le domaine d'application (Fig. 9). Ce film adhésif permettra, en prenant certaines précautions, le fonctionnement de l'appareil dans le cas de mesures de produits corrosifs. Il suffira ensuite de réaliser un nouvel étalonnage à l'aide de la banque d'échantillons.

\section{Conclusion}

Nous venons de présenter un appareillage inédit, permettant la mesure de constantes diélectriques complexes. Ce système présente de nombreux attraits, tels que :

- la fidélité et la sensiblité de la mesure concernant $\varepsilon^{\prime}$; 
— un coût modéré de l'investissement en matériel ;

- la possibilité pour tout opérateur, même inexpérimenté, d'effectuer des mesures, le logiciel de traitement des données étant conversationnel et très facile à utiliser ; il permet d'accéder à des fonctions annexes comme, par exemple, l'impression des résultats et la constitution d'un fichier de mesure ;

- une calibration rapide, puisqu'il suffit d'appliquer les échantillons d'étalonnage contre la sonde pendant le temps de la mesure, soit 1s (10 mesures successives sont prises lors de l'initialisation); une nouvelle initialisation peut, de plus, être effectuée à tout moment ;
- une caractérisation réalisée en un temps très court $(<0,5 \mathrm{~s}$, calcul et acquisition des données comprises).

Ce dispositif peut être employé pour la caractérisation rapide de diélectriques inconnus, pour le contrôle des propriétés de matériaux en cours de fabrication (suivi de polymérisations, de réactions chimiques, etc...).

L'imprécision sur la détermination de $\varepsilon^{\prime \prime}$ sera corrigée prochainement par la réalisation d'une sonde imprimée de type micro-ruban (remplaçant la ligne coaxiale) et par un contrôle de l'amplitude de l'oscillation à l'aide d'une boucle de rétroaction.

\section{Bibliographie}

[1] WHINNERY et JAMIESON « Equivalent circuits for discontinuities in transmission lines ", Proc. I.R.E. vol. 32, fév. 1944.

[2] WHINNERY, JAMIESON et ROBBINS «Coaxiale line discontinuities ", Proc. I.R.E. vol. 32, nov. 1944.

[3] TANABE E. et JoInes W. T. "A nondestructive method for measuring the complex permittivity of dielectric materials at microwave frequencies using an open transmission line resonator ", IEEE Trans. instrum. measur. vol. IM-25, $\mathrm{n}^{\circ}$ 3, sept. 1976.
[4] IDRISS I., "Contribution à la mesure de la constante diélectrique en hyper-fréquences ", Thèse INT Toulouse, 1979.

[5] voN Hippel A. R. « Les diélectriques et leurs applications ", (Dunod) 1961.

[6] Pelletier J.P. «Techniques numériques appliquées au calcul scientifique " (Masson) 1971.

[7] Référence technique IBM-PC XT, 1983.

[8] Rhode U. L. " Digital PLL frequency synthesizers, theory and design " (Prentice-Hall, Inc., Englewood Cliffs, N.J., 07632). 